\title{
靛红杂合喹唑啉类化合物的合成及抗肿瘤活性研究
}

\author{
张颖吕梦娇张娅玲陈丽王伟* 李宝林* \\ (教育部药用资源与天然药物化学重点实验室 陕西师范大学化学化工学院 西安 710062)
}

\begin{abstract}
摘要 合成了 9 种靛红杂合的喹唑啉类目标化合物, 借助 NMR、IR 和 HRMS 对目标化合物进行了结构表征, 并通过晶 体的培养及 X 射线衍射数据进一步确定了目标化合物的结构. 同时采用噻唑蓝(MTT)法在人结直肠癌细胞 SW480、人 非小细胞肺癌细胞 A549 和 NCI-H1975、人表皮鳞癌细胞 A431 上对这些化合物进行了抗肿瘤活性的初步体外评价. 结 果表明, 大部分目标化合物具有明显的抑制肿瘤细胞增殖的作用，尤其是化合物 $(E)-3-(((E)-(5-(4-(3-乙$ 乙炔苯胺基)喹唑 啉-6-基)呋喃-2-基)亚甲基)亚肼基)吲哚啉-2-酮(4a), 在 4 种所试肿瘤细胞上均表现出良好的抑制肿瘤细胞增殖的作用, 其效果优于临床使用的抗肿瘤药物拉帕替尼.
\end{abstract}

关键词 静红; 喹唑啉; 合成; 抗肿瘤活性

\section{Synthesis and Antitumor Activity of Heterozygous Isatin-Quinazoline Compounds}

\author{
Zhang, Ying Lü, Mengjiao Zhang, Yaling Chen, Li Wang, Wei* Li, Baolin*
}

(Key Laboratory of Ministry of Education for Medicinal Resources and Natural Pharmaceutical Chemistry, School of Chemistry \& Chemical Engineering, Shaanxi Normal University, Xi'an 710062)

\begin{abstract}
Nine new heterozygous isatin-quinazoline compounds were synthesized from cheap and easily available ortho nitrobenzaldehyde as the starting material. The chemical structures of the synthesized compounds were characterized by NMR,

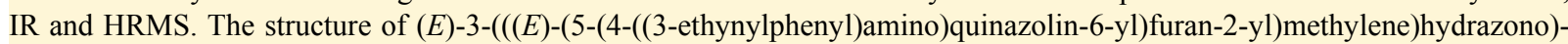
indolin-2-one (4a) was further determined by crystallization and X-ray diffraction, and the data revealed that its cis-trans isomerism was $(E, E)$. The antitumor activity of these new compounds was evaluated in vitro by methyl thiazolyl tetrazolium (MTT) assay in human colorectal carcinoma cells SW480, human non-small cell lung cancer cells A549 and NCI-H1975, and human epidermoid squamous carcinoma cells A431. The preliminary data demonstrated that most of the synthetic compounds had moderate to potent inhibitory activity against these four tumor cell lines. In particular, compound 4a had highly potent inhibitory activity on proliferation of four cell lines, and the activity was more potent than positive lapatinib.

Keywords isatin; quinazoline; synthesis; antitumor activity
\end{abstract}

近年来，以表皮生长因子受体(EGFR)酪氨酸激酶 为作用靶点的抗肿瘤治疗成为研究热点. 目前, 已经有 二十多种靶向酪氨酸激酶的小分子抑制剂. 例如, 吉非 替尼 ${ }^{[1]}$ 、埃罗替尼 ${ }^{[2,3]}$ 、拉帕替尼 ${ }^{[4,5]}$ 等已成功用于多种肿 瘤的临床治疗 ${ }^{[6]}$. 其中, 拉帕替尼(Lapatinib, 结构如 1) 由英国葛兰素史克公司研制开发. 它是一种可以口服 的、可逆的、小分子表皮生长因子受体 1 和 2 (ErbB1 和 ErbB2)抑制剂，可以同时竞争性结合 EGFR 和 HER2 胞内激酶区域的 ATP 结合位点, 从而抑制癌细胞的增 殖. 体内外的临床试验证明, Lapatinib 对治疗 EGFR 过
度表达引起的晚期或转移性乳腺癌、胃癌、非小细胞肺 癌以及结肠癌和膀胱癌均具有较好的疗效, 并且 Lapatinib 可明显延长患者的生存时间. 但是在使用过程 中发现，它仍存在着许多不良反应，包括腹泻、恶心、 呕吐、皮疹和肝损伤, 个别患者出现左室射血分数下降 (可逆的且可以导致呼吸短促)、间质性肺炎 ${ }^{[7]}$ 等副作用.

靛红(Isatin，结构如 2)及其衍生物具有广泛的药理 活性，对靛红可进行全方位的结构修饰，包括苯环的取 代, 1-N 取代及 2-C 和 3-C 的衍生, 其中以 3-C 上的结构 修饰最丰富, 其数量最多. 在 3-C 衍生得到的靛红类化

\footnotetext{
* Corresponding authors. E-mail: wwlzz@snnu.edu.cn; baolinli@snnu.edu.cn

Received January 18, 2017; revised February 20, 2017; published online March 8, 2017.

Project supported by the National Natural Science Foundation of China (No. 21272144)

国家自然科学基金(No. 21272144)资助项目.
} 
合物的药理活性中突出表现为抗癌作用 ${ }^{[8,9]}$. 3-C 衍生得 到的靛红衍生物是丝氨酸/苏氨酸蛋白激酶和受体酪氨 酸激酶 $\left(\mathrm{RTK}_{\mathrm{S}}\right)$ 抑制剂. 激酶调节许多与细胞增长、分化 和死亡相关的胞内外信号通路, 它们功能的失调会导致 一系列生理异常, 最终导致癌症的发生 ${ }^{[10]}$. 例如, 舒尼 替尼(Sunitinib, 结构如 3 ) 是由辉瑞制药公司研发的一种 新型多靶向性的治疗肿瘤的口服药物, 2006 年被食品药 品监督管理局(FDA)批准上市, 广泛用于治疗肾细胞 癌、胃肠间质瘤、肺癌、肝癌等疾病 ${ }^{[11]}$. 由于靛红衍生 物的多样性及其广泛的药理活性, 因此, 含有靛红结构 单元的小分子化合物成为近年来抗肿瘤药物研究领域 的一大热点 ${ }^{[12]}$.

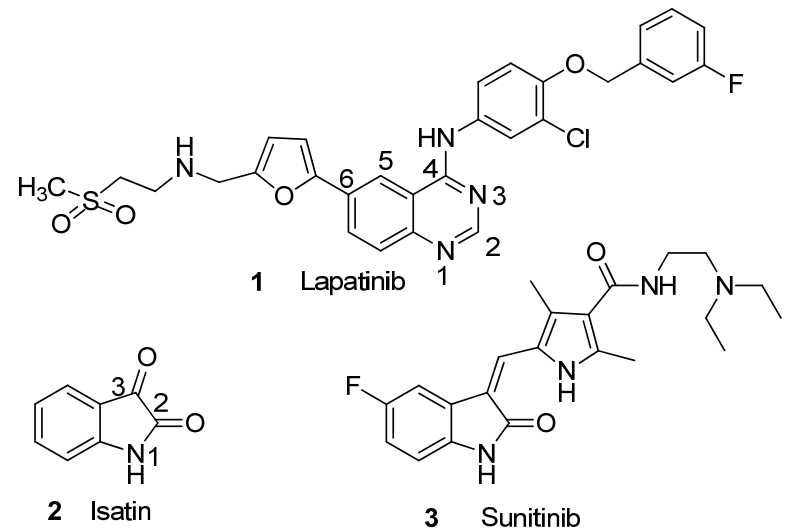

临床应用显示：一些针对某一靶标的药物使用一段 时间后, 会产生明显的耐药性, 给肿瘤的后期治疗带来 比较大的困难. 因此, 发现结构新颖、低毒、低耐药性 的抗肿瘤化合物仍是目前重要任务之一. 其中, 药效基 团的杂合是一种有效的、常用的合成新型药物的方法. 两个或两个以上的生物活性片段的杂合, 有互补的药效 功能或不同的作用机制，其表现出的协同作用可以有效 地治疗癌症 ${ }^{[13 ~ 15]}$. Fares 等 ${ }^{[16]}$ 将靛红与喹唑啉结构进行 杂合, 制备了一系列新颖的靛红衍生物, 其体外活性结 果显示: 部分化合物对人肝癌细胞(HepG2)的增殖有较 强的抑制活性. 基于以上启示, 本文在保持拉帕替尼药 效基团喹唑啉母核结构不变的基础上, 在其 6 位的呋喃 环上用 3 种不同的靛红衍生物替代原来的仲胺结构, 在 其 4 位保留拉帕替尼的芳胺基结构, 同时增加另外 2 种 不同的芳胺基结构单元, 共合成了 9 种结构新颖的靛红 杂合喹唑啉类化合物 $\mathbf{4 a} \sim \mathbf{4 i}$, 其合成路线如 Scheme 1 所示.

\section{1 结果与讨论}

\section{1 中间体及目标化合物的合成}

化合物 1 参考文献[17], 以邻硝基苯甲醛为起始原

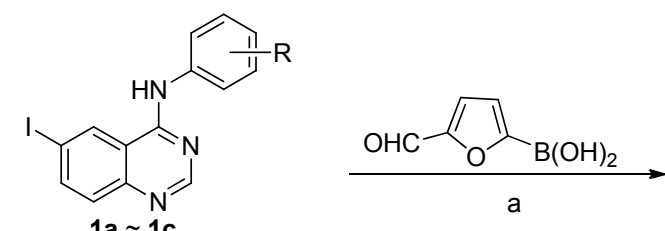

$1 \mathrm{a} \sim 1 \mathrm{c}$

1a: $R=3$-ethynyl;

1b: $\mathrm{R}=4-(E)$-propenyl

1c: $R=3$-chloro-4-(3-fluorobenzyloxy)

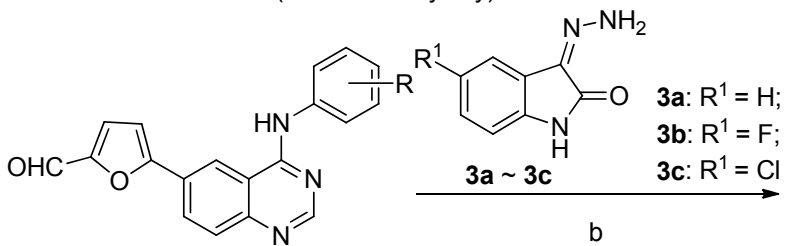

$2 \mathrm{a} \sim 2 \mathrm{c}$

2a: $R=3$-ethynyl;

2b: $\mathrm{R}=4-(E)$-propenyl;

2c: $R=3$-chloro-4-(3-fluorobenzyloxy)

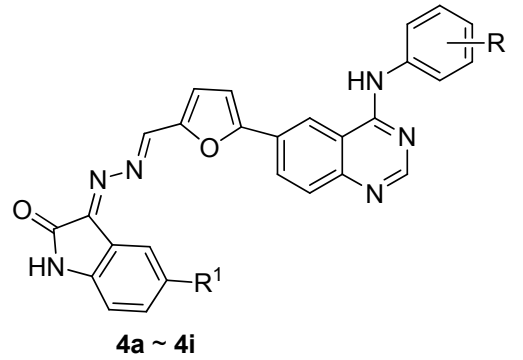

4a: $\mathrm{R}=$ 3-ethynyl, $\mathrm{R}^{1}=\mathrm{H} ; \mathbf{4 b}: \mathrm{R}=3$-ethynyl, $\mathrm{R}^{1}=\mathrm{F}$;

4c: $\mathrm{R}=3$-ethynyl, $\mathrm{R}^{1}=\mathrm{Cl} ; \mathbf{4 d}$ : $\mathrm{R}=4-(E)$-propenyl, $\mathrm{R}^{1}=\mathrm{H}$;

4e: $\mathrm{R}=4-(E)$-propenyl, $\mathrm{R}^{1}=\mathrm{F} ; \mathbf{4 f}: \mathrm{R}=4-(E)$-propenyl, $\mathrm{R}^{1}=\mathrm{Cl}$;

4g: $\mathrm{R}=3$-chloro-4-(3-fluorobenzyloxy), $\mathrm{R}^{1}=\mathrm{H}$;

4h: $R=3$-chloro-4-(3-fluorobenzyloxy), $R^{1}=F$;

4i: $\mathrm{R}=3$-chloro-4-(3-fluorobenzyloxy), $\mathrm{R}^{1}=\mathrm{Cl}$;

Reagents and conditions: (a) $5 \% \mathrm{Pd} / \mathrm{C}, \mathrm{DME}, \mathrm{MeOH}, \mathrm{Et}_{3} \mathrm{~N}, 50{ }^{\circ} \mathrm{C}$, $1.5 \mathrm{~h}$; (b): $\mathrm{C}_{2} \mathrm{H}_{5} \mathrm{OH}, \mathrm{CH}_{3} \mathrm{COOH}, \mathrm{DMF}, 80{ }^{\circ} \mathrm{C}, 6 \mathrm{~h}$

图式 1 目标化合物的合成路线

Scheme 1 Synthetic route for target compounds.

料，经过氰基化、硝基还原、碘代、成脒、Dimroth 重 排 6 步自行合成. 化合物 2 的合成采用 Suzuki 偶联

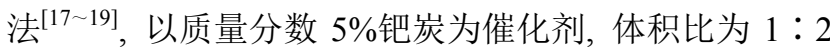
的甲醇与乙二醇二甲醚作混合溶剂，三乙胺为缚酸剂， 在 $50{ }^{\circ} \mathrm{C}$ 的条件下反应 $2 \sim 4 \mathrm{~h}$, 得到化合物 2. 该方法具 有反应时间较短，产率较高等优点. 化合物 3 的合成参

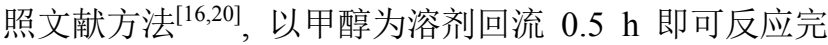
全，经过抽滤，重结晶，就可以直接用于下步反应。该 反应具有反应时间短，操作简单，后处理简单及收率高 等优点. 化合物 4 参照文献方法 $[21,22]$ 合成. 最初根据 文献[22]，用哌啶等弱碱作催化剂，催化该反应并没有 得到理想的产物. 后参考文献[21]用醋酸作催化剂, 乙 醇和 $N, N$-二甲基甲酰胺(DMF)作溶剂, $79{ }^{\circ} \mathrm{C}$ 反应 $6 \mathrm{~h}$. 反应完全，经抽滤、重结晶得到目标化合物. 该反应具 有操作简单，后处理容易等优点. 该反应是酸性催化的 
反应, 但不能用强酸, 因为氢离子与羰基结合可以增加 羰基亲电性的同时, 还可以和氨基结合, 这样丧失了胺 的亲核能力. 在弱酸醋酸的存在下, 一方面使羰基质子 化增强了其亲电性, 促进反应, 另一方面也避免了胺过 度质子化而造成亲核性下降.

\section{2 目标化合物 4 的结构确证}

目标化合物 4 的结构通过 NMR、IR 和 HRMS 确证. 现以化合物 $4 a$ 为例说明它们结构. 在化合物 $4 \mathbf{a}$ 的 ${ }^{1} \mathrm{H}$ NMR 谱中, $\delta 10.86(\mathrm{~s}, 1 \mathrm{H})$ 处出现 1 个单峰, 这是靛红 1 位 $\mathrm{NH}$ 的共振峰, $\delta 10.18(\mathrm{~s}, 1 \mathrm{H})$ 和 $9.04(\mathrm{~s}, 1 \mathrm{H})$ 处各出现 1 个单峰, 这是 4 位芳胺基喹唑啉类化合物的两个特征共 振信号, 前者是 4 位芳胺基 $\mathrm{NH}$ 的共振峰, 后者为喹唑 啉母核 2 位氢的共振峰. $\delta 8.68(\mathrm{~s}, 2 \mathrm{H})$ 处出现 1 个单峰, 分别是 $\mathrm{H}-\mathrm{C}=\mathrm{N}$ 的共振峰和喹唑啉母核 4 位氢的共振 峰, $\delta 8.36(\mathrm{~d}, J=8.7 \mathrm{~Hz}, 1 \mathrm{H})$ 和 $8.02(\mathrm{~d}, J=8.7 \mathrm{~Hz}, 1 \mathrm{H})$ 处 为喹坐啉母核 7, 8 位 2 个氢的共振峰, $\delta 7.60(\mathrm{~d}, J=3.6$ $\mathrm{Hz}, 1 \mathrm{H})$ 和 $7.49(\mathrm{~d}, J=3.6 \mathrm{~Hz}, 1 \mathrm{H})$ 处为呋喃环 2 个氢的共 振峰, $\delta 4.25(\mathrm{~s}, 1 \mathrm{H})$ 处为炔基氢的共振峰. 进一步通过 ${ }^{13} \mathrm{C} N M R 、 I R$ 和 HRMS 证明了化合物 $\mathbf{4 a}$ 是目标化合物. 在目标化合物中存在顺反构型, 为了进一步确定其构 型, 我们以 DMSO 为培养液, 对目标化合物进行单晶的 培养, 得到了化合物 $\mathbf{4 a}$ 的单晶, 结果表明其为 $(E, E)$ 构 型. 化合物 $4 \mathbf{a}$ 的晶体数据存于英国剑桥数据中心, CCDC 号为 1509375. 化合物 $\mathbf{4 a}$ 的晶体结构和分子结构 见图 1.

在该化合物的晶体结构中, 存在三种分子间氢键: $\mathrm{N}(3)-\mathrm{H}(3) \cdots \mathrm{O}(1), \mathrm{C}(10)-\mathrm{H}(10) \cdots \mathrm{O}(1), \mathrm{N}(6)-\mathrm{H}(6) \cdots$ $\mathrm{N}(1)$ (图 2), 键长分别为 2.936(2), 3.338(3), 2.854(2) $\AA$, 键角分别为 $\mathrm{N}(3)-\mathrm{H}(3) \cdots \mathrm{O}(1)=158^{\circ}, \mathrm{C}(10)-\mathrm{H}(10) \cdots$ $\mathrm{O}(1)=149^{\circ}, \mathrm{N} 6-\mathrm{H}(6) \cdots \mathrm{N}(1)=162^{\circ}$, 这些氢键在单晶 堆积过程中起到很重要的作用.
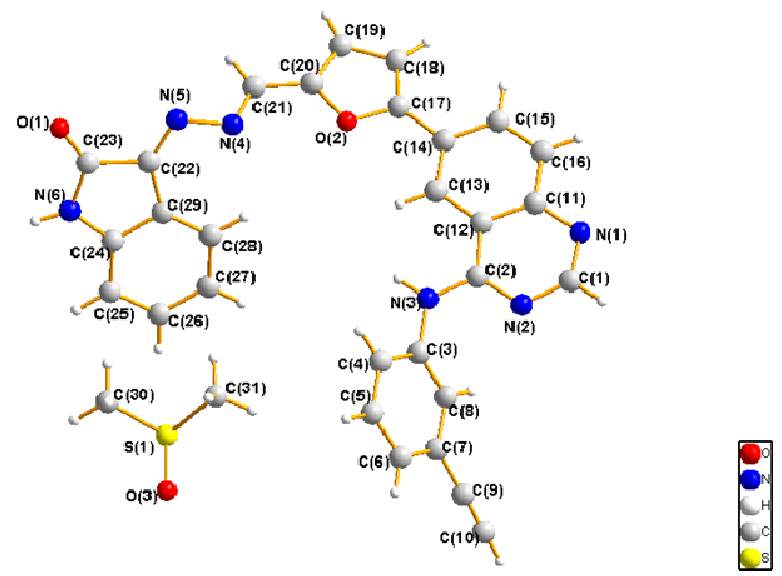

图 1 化合物 $4 \mathrm{a}$ 的分子结构和晶体结构

Figure 1 Chemical and crystal structure of compound $\mathbf{4 a}$

\section{3 抗肿瘤活性}

本文以临床抗肿瘤药物 Lapatinib 为阳性对照, 以 人结直肠癌细胞 SW 480 、人非小细胞肺癌细胞 A549 和 NCI-H1975、人表皮鳞癌细胞 A431 为受试细胞株, 用 噻唑蓝(MTT)法对所合成的新化合物 $\mathbf{4 a} \sim \mathbf{4 i}$ 进行了初步 的抗肿瘤活性测试, 其实验数据如表 1 所示. 实验数据 表明, 所合成化合物 $4 \mathrm{a} 、 4 \mathrm{~b} 、 4 \mathrm{c} 、 4 \mathrm{~g} 、 4 \mathrm{~h}$ 表现出较强的 抑制肿瘤细胞增殖活性. 受试化合物 $4 a 、 4 b 、 4 c 、 4 g 、$ $4 h$ 对 $\mathrm{A} 431$ 的增殖具有明显的抑制作用, 其 $\mathrm{IC}_{50}$ 值分别 为 $1.93 \pm 1.06,0.64 \pm 0.07,1.31 \pm 0.50,1.06 \pm 0.40,1.84 \pm$ $0.84 \mu \mathrm{mol} \cdot \mathrm{L}^{-1}$, 其效果优于阳性对照拉帕替尼 $(4.80 \pm$ $0.71 \mu \mathrm{mol} \cdot \mathrm{L}^{-1}$ ); 受试化合物 $4 \mathrm{a} 、 4 \mathbf{c} 、 4 \mathrm{~g}$ 对 $\mathrm{SW} 480$ 的增 殖抑制作用与阳性对照拉帕替尼相当, 受试化合物 4a、 4b、4c 对 NCI-H1975 的增殖具有明显的抑制作用, 受试 化合物 4a 对 A549 的增殖具有明显的抑制作用. 综上所 述, 化合物 $\mathbf{4 a}$ 对 4 种所试肿瘤细胞的增殖均表现出良好 的抑制作用，其活性均优于临床用药拉帕替尼. 根据实

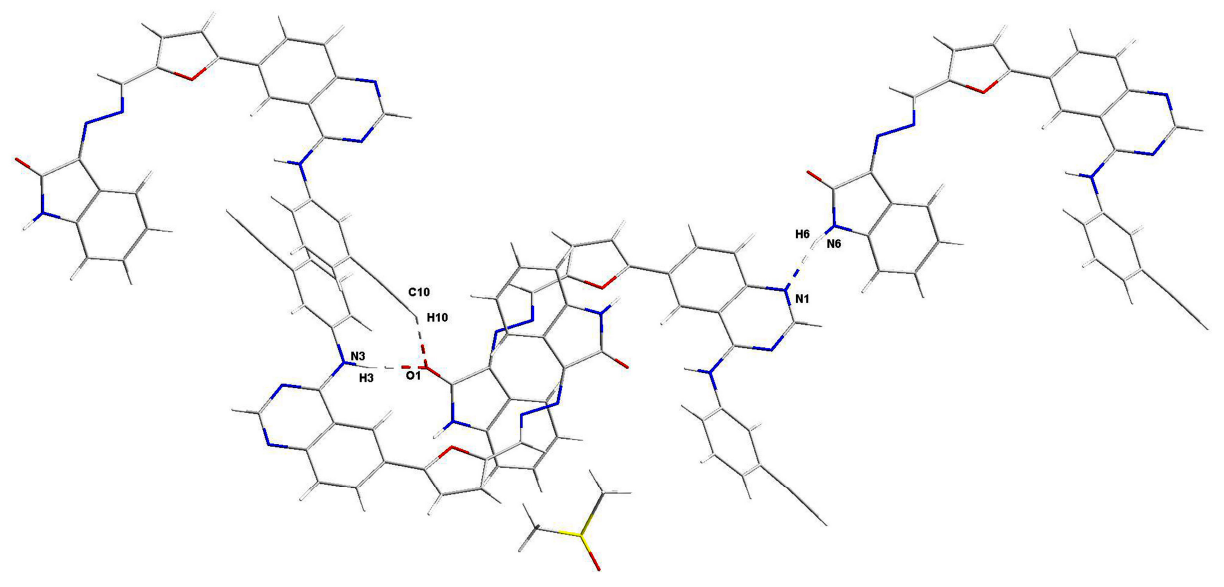

图 2 化合物 4a 中分子间氢键图

Figure 2 Hydrogen bonds in compound 4a 
表 1 目标化合物对 4 种受试细胞的体外抑制活性 $\left[\mathrm{IC}_{50} /\left(\mu \mathrm{mol} \cdot \mathrm{L}^{-1}\right)\right]^{a}$

Table 1 Inhibitory activity $\left[\mathrm{IC}_{50} /\left(\mu \mathrm{mol} \cdot \mathrm{L}^{-1}\right)\right]$ of target compounds against four tested cells growth in vitro

\begin{tabular}{cllll}
\hline Compound & SW480 & \multicolumn{1}{c}{ A549 } & A431 & NCI-H1975 \\
\hline Lapatinib & $12.58 \pm 1.35$ & $14.90 \pm 1.21$ & $4.80 \pm 0.71$ & $12.68 \pm 0.73$ \\
Isatin & $>100$ & $>100$ & $>100$ & $>100$ \\
$\mathbf{4 a}$ & $14.86 \pm 1.08$ & $10.15 \pm 3.91$ & $1.93 \pm 1.06$ & $11.54 \pm 1.29$ \\
$\mathbf{4 b}$ & $>100$ & $>100$ & $0.64 \pm 0.07$ & $12.85 \pm 1.85$ \\
$\mathbf{4}$ & $8.44 \pm 4.64$ & $22.72 \pm 10.53$ & $1.31 \pm 0.50$ & $5.77 \pm 1.43$ \\
$\mathbf{4 d}$ & $>100$ & $>100$ & $33.67 \pm 6.87$ & $32.67 \pm 0.50$ \\
$\mathbf{4}$ & $>100$ & $>100$ & $105.90 \pm 40.50$ & $>100$ \\
$\mathbf{4}$ & $>100$ & $>100$ & $>100$ & $>100$ \\
$\mathbf{4 g}$ & $15.43 \pm 3.54$ & $>100$ & $1.06 \pm 0.40$ & $16.70 \pm 0.36$ \\
$\mathbf{4 h}$ & $89.41 \pm 15.68$ & $>100$ & $1.84 \pm 0.84$ & $143.10 \pm 22.91$ \\
$\mathbf{4 i}$ & $>100$ & $>100$ & $>100$ & $>100$ \\
\hline
\end{tabular}

${ }^{a} \mathrm{IC}_{50}$ values are shown as the mean $\pm \mathrm{SD}\left(\mu \mathrm{mol} \bullet \mathrm{L}^{-1}\right)$ from three independent experiments.

验数据可知: 当喹唑啉母核 4 位为 3-乙炔苯胺基时, 抑 制肿瘤细胞增殖活性最好; 当 4 位为 3-氯-4-(3-氟苄氧 基)苯胺基时, 活性次之; 当 4 位为 4-(E)-丙烯基苯胺基 时, 对肿瘤细胞增殖的抑制不明显. 靛红 5 位无取代基 时，抑制肿瘤细胞增殖活性最好.

\section{2 结论}

本文经 8 步反应合成了 9 种结构新颖的拉帕替尼类 似物, 其反应原料易得, 操作步骤简单. 利用 NMR、IR 和 HRMS 波谱学方法对反应中间体及目标化合物结构 进行了确证, 并通过 X 射线衍射对其构型进行了确认. 另外以 4 种人肿瘤细胞为模型, 采用 MTT 法对目标化 合物进行了初步的体外抗肿瘤活性评价. 测试结果表 明，化合物 $4 \mathrm{a} 、 4 \mathrm{~b} 、 4 \mathrm{c} 、 4 \mathrm{~g} 、 4 \mathrm{~h}$ 表现出较强的抑制肿瘤 细胞增殖活性. 化合物 4b 对 A431 和 NCI-H1975 的增殖 具有明显的抑制作用, 化合物 $\mathbf{4 g}$ 对 SW480 和 A431 的 增殖具有明显的抑制作用, 化合物 $4 h$ 对 A431 的增殖具 有明显的抑制作用, 化合物 4c 对 SW480、A431 和 NCI-H1975 的增殖具有明显的抑制作用, 尤其是化合物 4a 在 4 种所试肿瘤细胞上均表现出良好的抑制肿瘤细 胞增殖活性, 优于临床使用的抗肿瘤药物拉帕替尼 (Lapatinib), 这预示着通过对化合物 $\mathbf{4 a}$ 的进一步研究, 有望开发出新的具有抗肿瘤活性的药物.

\section{3 实验部分}

\section{1 仪器与试剂}

核磁共振数据由 Bruker Avance 300、400、600 超导 核磁共振仪测定, TMS 作为内标; 红外光谱数据采用 Nicolet 170SX FT-IR 红外光谱仪测定; 质谱数据用 Bruker Esquire 3000plus 质谱仪测定; 熔点采用 X-6 显微 熔点测定仪(北京泰克仪器有限公司)测定(温度未进行 校正); 晶体数据由德国 Bruker APEX-II CCD 单晶 X 射
线衍射仪在 $153 \mathrm{~K}$ 温度下收集数据. 人结直肠癌细胞 SW480、人非小细胞肺癌细胞 A549 和 NCI-H1975、人 表皮鳞癌细胞 A431 均购自中国科学院细胞库. 所用试 剂均为分析纯.

\section{2 实验方法}

\subsection{1 化合物 $\mathbf{2}$ 的合成}

取 $6.0 \mathrm{mmol}$ 化合物 $\mathbf{1}, 1.12 \mathrm{~g}(8.0 \mathrm{mmol}) 5$-甲酰基呋 喃-2-硼酸、0.56 g 5\%钯炭, $40 \mathrm{~mL}$ 乙二醇二甲醚、 $20 \mathrm{~mL}$ 甲醇和 $2.42 \mathrm{~g}(24 \mathrm{mmol})$ 三乙胺加入到反应瓶中, $50{ }^{\circ} \mathrm{C}$ 搅拌, TLC 跟踪(展开剂 $V_{\text {乙配醌 }}: V_{\text {氛仿 }}=1: 3$ ) 至反应完 毕, 约需反应时间 $3 \mathrm{~h}$. 趁热抽滤, 减压旋蒸滤液, 所得 残留物进行硅胶柱色谱分离 $(V$ 乙配怔： $V$ 氯仿 $=1: 9)$ 得到 橘黄色固体 2 .

4-(3-乙炔基苯胺基)-6-(5-甲酰基呋喃-2-基)喹唑啉 (2a) ${ }^{[18]}$ : m.p. $247.8 \sim 249.3{ }^{\circ} \mathrm{C}$. 橘黄色固体, $1.55 \mathrm{~g}$, 收率 为 $76.3 \%$.

4-[4-(E)-丙烯基苯胺基]-6-(5-甲酰基呋喃-2-基)喹唑 啉 $(\mathbf{2 b})^{[18]}$ : m.p. $230.1 \sim 231.9{ }^{\circ} \mathrm{C}$. 橘黄色固体, $1.23 \mathrm{~g}$, 收率为 $57.7 \%$.

4-[3-氯-4-(3-氟苄氧基)苯胺基]-6-(5-甲酰基呋喃-2基)喹唑啉(2c): m.p. $229.6 \sim 231.4{ }^{\circ} \mathrm{C}$ (文献值 ${ }^{[17]}$ : m.p. $229 \sim 233{ }^{\circ} \mathrm{C}$ ). 橘黄色固体, $2.46 \mathrm{~g}$, 收率为 $86.6 \%$.

\subsection{2 化合物 4 的合成}

取 $0.5 \mathrm{mmol}$ 化合物 2、0.5 mmol 化合物 3、0.5 mL

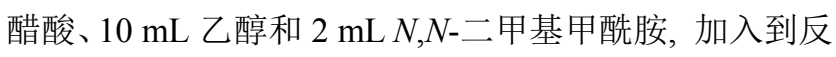
应瓶中, $80{ }^{\circ} \mathrm{C}$ 回流, TLC 跟踪(展开剂 $V_{\text {乙配的 }}: V$ 氯仿 $=$ $1: 1$ )至反应完毕, 约需反应时间 $6 \mathrm{~h}$. 冷却后抽滤, 用 乙醇冲洗滤饼, 滤饼中的固体用二甲亚砜重结晶, 析出 大量红色固体 4.

$(E)$-3-(((E)-(5-(4-(3-乙炔苯胺基)喹唑啉-6-基)呋喃2-基)亚甲基)亚肼基)吲哚啉-2-酮(4a)：红色固体 $0.18 \mathrm{~g}$, 收率为 $73.5 \%$. m.p. $>280{ }^{\circ} \mathrm{C} ;{ }^{1} \mathrm{H}$ NMR $(600 \mathrm{MHz}$, 
DMSO- $\left.d_{6}\right) \delta: 10.86(\mathrm{~s}, 1 \mathrm{H}), 10.18(\mathrm{~s}, 1 \mathrm{H}), 9.04(\mathrm{~s}, 1 \mathrm{H})$, $8.68(\mathrm{~s}, 2 \mathrm{H}), 8.36(\mathrm{~d}, J=8.7 \mathrm{~Hz}, 1 \mathrm{H}), 8.35(\mathrm{~d}, J=7.9 \mathrm{~Hz}$, 1H), 8.07 (s, 1H), 8.02 (d, $J=8.7 \mathrm{~Hz}, 1 \mathrm{H}), 7.93$ (d, $J=8.7$ $\mathrm{Hz}, 1 \mathrm{H}), 7.60(\mathrm{~d}, J=3.6 \mathrm{~Hz}, 1 \mathrm{H}), 7.49$ (d, $J=3.6 \mathrm{~Hz}, 1 \mathrm{H})$, 7.46 (t, $J=7.9 \mathrm{~Hz}, 1 \mathrm{H}), 7.39$ (t, $J=7.4 \mathrm{~Hz}, 1 \mathrm{H}), 7.28$ (d, $J=7.6 \mathrm{~Hz}, 1 \mathrm{H}), 7.07(\mathrm{t}, J=7.6 \mathrm{~Hz}, 1 \mathrm{H}), 6.90(\mathrm{~d}, J=7.8$ $\mathrm{Hz}, 1 \mathrm{H}), 4.25(\mathrm{~s}, 1 \mathrm{H}) ;{ }^{13} \mathrm{C}$ NMR (101 MHz, DMSO- $\left.d_{6}\right) \delta$ : $165.2,158.2,157.2,155.4,152.1,151.9,150.4,149.7$, $145.3,139.8,134.1,130.1,129.8,129.4,129.3,127.3$, $127.2,125.3,123.1,122.9,122.8,122.3,119.5,117.2$, 116.0, 111.1, 111.0, 83.8, 81.1; IR (KBr) v: 3545, 3417, $3235,2062,1639,1617,1487,1396,1174,623 \mathrm{~cm}^{-1}$; HRMS calcd for $\mathrm{C}_{29} \mathrm{H}_{19} \mathrm{~N}_{6} \mathrm{O}_{2}[\mathrm{M}+\mathrm{H}]^{+}: 483.1569$, found 483.1581 .

(E)-3-(((E)-(5-(4-(3-乙炔苯胺基)喹唑啉-6-基)呋喃2-基)亚甲基)亚肼基)-5-氟吲哚啉-2-酮(4b): 红色固体 $0.18 \mathrm{~g}$, 收率为 $72.6 \%$. m.p. $>280{ }^{\circ} \mathrm{C} ;{ }^{1} \mathrm{H}$ NMR $(400$ MHz, DMSO- $\left.d_{6}\right) \delta: 10.83(\mathrm{~s}, 1 \mathrm{H}), 10.05(\mathrm{~s}, 1 \mathrm{H}), 9.00$ (s, $1 \mathrm{H}), 8.67(\mathrm{~s}, 1 \mathrm{H}), 8.63(\mathrm{~s}, 1 \mathrm{H}), 8.26(\mathrm{~d}, J=8.7 \mathrm{~Hz}, 1 \mathrm{H})$, 8.07 (d, $J=9.2 \mathrm{~Hz}, 2 \mathrm{H}), 7.95$ (d, $J=8.7 \mathrm{~Hz}, 1 \mathrm{H}), 7.85$ (d, $J=8.6 \mathrm{~Hz}, 1 \mathrm{H}), 7.58(\mathrm{~d}, J=3.3 \mathrm{~Hz}, 1 \mathrm{H}), 7.42(\mathrm{t}, J=7.8$ $\mathrm{Hz}, 2 \mathrm{H}), 7.26(\mathrm{~d}, J=7.9 \mathrm{~Hz}, 1 \mathrm{H}), 7.21(\mathrm{~d}, J=8.7 \mathrm{~Hz}, 1 \mathrm{H})$, $6.86(\mathrm{dd}, J=8.4,4.1 \mathrm{~Hz}, 1 \mathrm{H}), 4.20(\mathrm{~s}, 1 \mathrm{H}) ;{ }^{13} \mathrm{C}$ NMR $(151$ MHz, DMSO- $\left.d_{6}\right) \delta: 164.8,158.4,157.7,157.0,155.9(\mathrm{~d}$, $\left.{ }^{1} J_{\mathrm{C}-\mathrm{F}}=277.9 \mathrm{~Hz}\right), 152.3,151.6\left(\mathrm{~d},{ }^{4} J_{\mathrm{C}-\mathrm{F}}=2.5 \mathrm{~Hz}\right), 150.0$, $149.1,141.1,139.2,129.0,128.9,128.8,127.0,126.7$, $125.2,122.9,121.8,120.0,119.9,119.2,117.2\left(\mathrm{~d},{ }^{3} J_{\mathrm{C}-\mathrm{F}}=\right.$ $9.2 \mathrm{~Hz}), 116.2\left(\mathrm{~d},{ }^{2} J_{\mathrm{C}-\mathrm{F}}=26.4 \mathrm{~Hz}\right), 115.4\left(\mathrm{~d},{ }^{2} J_{\mathrm{C}-\mathrm{F}}=12.5\right.$ $\mathrm{Hz}), 111.6\left(\mathrm{~d},{ }^{3} J_{\mathrm{C}-\mathrm{F}}=7.7 \mathrm{~Hz}\right), 110.6,83.4,80.6$; IR $(\mathrm{KBr}) v$ : $3563,3416,3235,2061,1718,1698,1616,1519,1035$, $624 \mathrm{~cm}^{-1}$; HRMS calcd for $\mathrm{C}_{29} \mathrm{H}_{18} \mathrm{FN}_{6} \mathrm{O}_{2}[\mathrm{M}+\mathrm{H}]^{+}$: 501.1475, found 501.1491.

(E)-3-(((E)-(5-(4-(3-乙炔苯胺基)喹唑啉-6-基)呋喃2-基)亚甲基)亚肼基)-5-氯吲哚啉-2-酮(4c): 红色固体 $0.20 \mathrm{~g}$, 收率为 $76.3 \%$. m.p. $>280{ }^{\circ} \mathrm{C} ;{ }^{1} \mathrm{H}$ NMR $(600$ MHz, DMSO- $\left.d_{6}\right) \delta: 10.90(\mathrm{~s}, 1 \mathrm{H}), 9.96(\mathrm{~s}, 1 \mathrm{H}), 8.93$ (s, $1 \mathrm{H}), 8.64$ (s, 1H), 8.59 (s, 1H), 8.33 (s, 1H), 8.24 (d, $J=$ $8.7 \mathrm{~Hz}, 1 \mathrm{H}), 8.00(\mathrm{~s}, 1 \mathrm{H}), 7.90(\mathrm{~d}, J=8.0 \mathrm{~Hz}, 1 \mathrm{H}), 7.78$ (d, $J=8.7 \mathrm{~Hz}, 1 \mathrm{H}), 7.50(\mathrm{~s}, 1 \mathrm{H}), 7.42(\mathrm{t}, J=7.9 \mathrm{~Hz}, 1 \mathrm{H})$, $7.38 \sim 7.36(\mathrm{~m}, 2 \mathrm{H}), 7.26(\mathrm{~d}, J=7.4 \mathrm{~Hz}, 1 \mathrm{H}), 6.84$ (d, $J=$ $8.2 \mathrm{~Hz}, 1 \mathrm{H}), 4.20(\mathrm{~s}, 1 \mathrm{H}) ;{ }^{13} \mathrm{C}$ NMR $\left(101 \mathrm{MHz}\right.$, DMSO- $\left.d_{6}\right)$ $\delta: 164.5,157.7,156.9,155.0,152.5,151.6,149.9,149.0$, $143.4,139.2,132.8,129.1,128.9,128.6,127.1,126.6$, $126.0,125.4,123.6,123.5,123.1,121.7,119.3,118.0$, 115.4, 112.0, 110.4, 83.4, 80.7; IR (KBr) v: 3555, 3416,
3235, 2062, 1719, 1638, 1616, 1396, 1174, $623 \mathrm{~cm}^{-1}$; HRMS calcd for $\mathrm{C}_{29} \mathrm{H}_{18} \mathrm{ClN}_{6} \mathrm{O}_{2}[\mathrm{M}+\mathrm{H}]^{+}: 517.1180$, found 517.1182 .

(E)-3-(((E)-(5-(4-(4- $(E)$-丙烯基苯胺基)喹唑啉-6-基) 呋喃-2-基)亚甲基)亚肼基)吲哚啉-2-酮(4d)：红色固体 $0.18 \mathrm{~g}$, 收率为 72.1\%. m.p. $>280{ }^{\circ} \mathrm{C} ;{ }^{1} \mathrm{H}$ NMR $(300$ MHz, DMSO- $\left.d_{6}\right) \delta: 10.85(\mathrm{~s}, 1 \mathrm{H}), 10.08(\mathrm{~s}, 1 \mathrm{H}), 8.98(\mathrm{~s}$, $1 \mathrm{H}), 8.64(\mathrm{~s}, 1 \mathrm{H}), 8.60(\mathrm{~s}, 1 \mathrm{H}), 8.30(\mathrm{~d}, J=8.0 \mathrm{~Hz}, 2 \mathrm{H})$, $7.87(\mathrm{~d}, J=8.7 \mathrm{~Hz}, 1 \mathrm{H}), 7.81(\mathrm{~d}, J=8.4 \mathrm{~Hz}, 2 \mathrm{H}), 7.56(\mathrm{~d}, J$ $=3.3 \mathrm{~Hz}, 1 \mathrm{H}), 7.43 \sim 7.40(\mathrm{~m}, 3 \mathrm{H}), 7.36(\mathrm{~d}, J=7.5 \mathrm{~Hz}$, 1H), 7.04 (t, $J=7.5 \mathrm{~Hz}, 1 \mathrm{H}), 6.90$ (d, $J=7.8 \mathrm{~Hz}, 1 \mathrm{H}), 6.41$ (d, $J=15.9 \mathrm{~Hz}, 1 \mathrm{H}), 6.25(\mathrm{dq}, J=15.9,6.1 \mathrm{~Hz}, 1 \mathrm{H}), 1.86$ $(\mathrm{d}, J=6.1 \mathrm{~Hz}, 3 \mathrm{H}) ;{ }^{13} \mathrm{C}$ NMR $\left(151 \mathrm{MHz}, \mathrm{DMSO}-d_{6}\right) \delta$ : $164.8,157.7,156.7,154.9,151.6,151.1,149.6,149.2$, $144.9,137.6,133.6,133.3,130.4,129.6,129.1,128.5$, $126.7,125.8,124.5,122.7,122.5,122.0,119.1,116.8$, $115.5,110.6,110.4,18.2$; IR (KBr) v: 3416, 3001, 2062, $1617,1518,1487,1397,1175,787,622 \mathrm{~cm}^{-1}$; HRMS calcd for $\mathrm{C}_{30} \mathrm{H}_{23} \mathrm{~N}_{6} \mathrm{O}_{2}[\mathrm{M}+\mathrm{H}]^{+}:$499.1882, found 499.1900.

(E)-3-(( $(E)-(5-(4-(4-(E)$-丙烯基苯胺基)喹唑啉-6-基) 呋喃-2-基)亚甲基)亚肼基)-5-氟吲哚啉-2-酮(4e)：红色 固体 $0.20 \mathrm{~g}$, 收率为 $75.8 \%$. m.p. $>280{ }^{\circ} \mathrm{C} ;{ }^{1} \mathrm{H}$ NMR $(600$ $\left.\mathrm{MHz}, \mathrm{DMSO}-d_{6}\right) \delta:{ }^{1} \mathrm{H}$ NMR (600 MHz, DMSO- $\left.d_{6}\right) \delta$ : 10.84 (s, 1H), 10.00 (s, 1H), 9.00 (s, 1H), 8.67 (s, 1H), $8.58(\mathrm{~s}, 1 \mathrm{H}), 8.25(\mathrm{dd}, J=8.5,1.2 \mathrm{~Hz}, 1 \mathrm{H}), 8.07$ (d, $J=8.7$ $\mathrm{Hz}, 1 \mathrm{H}), 7.84$ (d, $J=8.7 \mathrm{~Hz}, 1 \mathrm{H}), 7.81$ (d, $J=8.4 \mathrm{~Hz}, 2 \mathrm{H})$, 7.59 (d, $J=3.6 \mathrm{~Hz}, 1 \mathrm{H}), 7.42(\mathrm{~d}, J=3.6 \mathrm{~Hz}, 1 \mathrm{H}), 7.40$ (d, $J=8.4 \mathrm{~Hz}, 2 \mathrm{H}), 7.25(\mathrm{td}, J=9.0,2.7 \mathrm{~Hz}, 1 \mathrm{H}), 6.89(\mathrm{dd}$, $J=8.5,4.2 \mathrm{~Hz}, 1 \mathrm{H}), 6.41(\mathrm{~d}, J=15.7 \mathrm{~Hz}, 1 \mathrm{H}), 6.26(\mathrm{dq}$, $J=15.7,6.5 \mathrm{~Hz}, 1 \mathrm{H}), 1.86(\mathrm{~d}, J=6.5 \mathrm{~Hz}, 3 \mathrm{H}) ;{ }^{13} \mathrm{C} \mathrm{NMR}$ $\left(151 \mathrm{MHz}, \mathrm{DMSO}-d_{6}\right) \delta: 164.8,158.4,157.6,157.1,155.9$ $\left(\mathrm{d},{ }^{1} J_{\mathrm{C}-\mathrm{F}}=258.1 \mathrm{~Hz}\right), 152.3,151.6,149.9,149.1,141.2$, 137.6, 133.2, 130.4, 128.8, 128.7, 126.6, 125.7, 124.5, $122.8,122.5,120.0,119.9\left(\mathrm{~d},{ }^{2} J_{\mathrm{C}-\mathrm{F}}=23.8 \mathrm{~Hz}\right), 117.2(\mathrm{~d}$, $\left.{ }^{3} J_{\mathrm{C}-\mathrm{F}}=9.1 \mathrm{~Hz}\right), 116.2\left(\mathrm{~d},{ }^{2} J_{\mathrm{C}-\mathrm{F}}=25.7 \mathrm{~Hz}\right), 115.5,111.6(\mathrm{~d}$, $\left.{ }^{3} J_{\mathrm{C}-\mathrm{F}}=8.1 \mathrm{~Hz}\right), 110.5,18.2$; IR (KBr) $v: 3417,2986,2062$, 1617, 1486, 1397, 1173, 1003, 787, $622 \mathrm{~cm}^{-1}$; HRMS calcd for $\mathrm{C}_{30} \mathrm{H}_{22} \mathrm{FN}_{6} \mathrm{O}_{2}[\mathrm{M}+\mathrm{H}]^{+}:$517.1788, found 517.1795.

$(E)-3-(((E)-(5-(4-(4-(E)$-丙烯基苯胺基)喹唑啉-6-基) 呋喃-2-基)亚甲基)亚肼基)-5-氯吲哚啉-2-酮(4f): 红色固 体 $0.21 \mathrm{~g}$, 收率为 79.3\%. m.p. $>280{ }^{\circ} \mathrm{C} ;{ }^{1} \mathrm{H}$ NMR $(600$ MHz, DMSO- $\left.d_{6}\right) \delta: 10.96(\mathrm{~s}, 1 \mathrm{H}), 10.03(\mathrm{~s}, 1 \mathrm{H}), 9.04$ (s, $1 \mathrm{H}), 8.71(\mathrm{~s}, 1 \mathrm{H}), 8.59(\mathrm{~s}, 1 \mathrm{H}), 8.41(\mathrm{~s}, 1 \mathrm{H}), 8.32(\mathrm{~d}, J=$ 
$7.7 \mathrm{~Hz}, 1 \mathrm{H}), 7.85(\mathrm{~d}, J=8.2 \mathrm{~Hz}, 1 \mathrm{H}), 7.78(\mathrm{~d}, J=6.7 \mathrm{~Hz}$, 2H), $7.58(\mathrm{~s}, 1 \mathrm{H}), 7.43 \sim 7.38(\mathrm{~m}, 4 \mathrm{H}), 6.91(\mathrm{~d}, J=8.0 \mathrm{~Hz}$, 1H), 6.43 (d, $J=15.8 \mathrm{~Hz}, 1 \mathrm{H}), 6.28$ (dd, $J=15.8,5.6 \mathrm{~Hz}$, $1 \mathrm{H}), 1.87(\mathrm{~d}, J=5.6 \mathrm{~Hz}, 3 \mathrm{H}) ;{ }^{13} \mathrm{C}$ NMR $(101 \mathrm{MHz}$, DMSO- $\left.d_{6}\right) \delta: 164.5,157.7,157.0,155.1,152.5,151.6$, $149.8,148.9,143.5,137.5,133.2,132.8,130.4,129.1$, $128.7,128.5,126.5,126.0,125.7,124.5,123.6,122.7$, 119.4, 118.0, 115.5, 112.1, 110.4, 18.3; IR (KBr) v: 3448, 3409, 2990, 1722, 1614, 1515, 1394, 1178, 786, $624 \mathrm{~cm}^{-1}$; HRMS calcd for $\mathrm{C}_{30} \mathrm{H}_{22} \mathrm{ClN}_{6} \mathrm{O}_{2}[\mathrm{M}+\mathrm{H}]^{+}: 533.1493$, found 533.1509 .

$(E)-3-(((E)-(5-(4-(3-$ 氯-4-(3-氟苄氧基)苯胺基)喹唑 啉-6-基)呋喃-2-基)亚甲基)亚肼基)吲哚啉-2-酮(4g)：红 色固体 $0.21 \mathrm{~g}$, 收率为 $69.3 \%$. m.p. $>280{ }^{\circ} \mathrm{C} ;{ }^{1} \mathrm{H}$ NMR $\left(600 \mathrm{MHz}, \mathrm{DMSO}-d_{6}\right) \delta: 10.85(\mathrm{~s}, 1 \mathrm{H}), 10.07(\mathrm{~s}, 1 \mathrm{H}), 8.97$ (s, 1H), $8.65(\mathrm{~s}, 1 \mathrm{H}), 8.61(\mathrm{~s}, 1 \mathrm{H}), 8.32 \sim 8.30(\mathrm{~m}, 2 \mathrm{H})$, $8.03(\mathrm{~d}, J=2.4 \mathrm{~Hz}, 1 \mathrm{H}), 7.88(\mathrm{~d}, J=8.9 \mathrm{~Hz}, 1 \mathrm{H}), 7.79(\mathrm{dd}$, $J=8.9,2.4 \mathrm{~Hz}, 1 \mathrm{H}), 7.57(\mathrm{~d}, J=3.6 \mathrm{~Hz}, 1 \mathrm{H}), 7.50 \sim 7.46$ (m, 1H), $7.43(\mathrm{~d}, J=3.6 \mathrm{~Hz}, 1 \mathrm{H}), 7.36 \sim 7.33(\mathrm{~m}, 3 \mathrm{H})$, 7.29 (d, $J=9.0 \mathrm{~Hz}, 1 \mathrm{H}), 7.19$ (t, $J=8.5 \mathrm{~Hz}, 1 \mathrm{H}), 7.04$ (t, $J=7.5 \mathrm{~Hz}, 1 \mathrm{H}), 6.90(\mathrm{~d}, J=7.7 \mathrm{~Hz}, 1 \mathrm{H}), 5.27(\mathrm{~s}, 2 \mathrm{H}) ;{ }^{13} \mathrm{C}$ NMR $\left(151 \mathrm{MHz}\right.$, DMSO- $\left.d_{6}\right) \delta: 165.3,162.7\left(\mathrm{~d},{ }^{1} J_{\mathrm{C}-\mathrm{F}}=\right.$ $243.8 \mathrm{~Hz}), 158.2,157.3,155.5,152.1,151.7,150.4,150.3$, $149.7,145.4,140.1\left(\mathrm{~d},{ }^{3} J_{\mathrm{C}-\mathrm{F}}=7.5 \mathrm{~Hz}\right), 134.1,133.6,131.0$ $\left(\mathrm{d},{ }^{3} J_{\mathrm{C}-\mathrm{F}}=8.3 \mathrm{~Hz}\right), 130.2,129.7,129.3,127.2,124.5,123.8$ $\left(\mathrm{d},{ }^{4} J_{\mathrm{C}-\mathrm{F}}=2.5 \mathrm{~Hz}\right), 122.9,122.7,121.6,119.4,117.3,115.9$, $115.2\left(\mathrm{~d},{ }^{2} J_{\mathrm{C}-\mathrm{F}}=20.8 \mathrm{~Hz}\right), 114.8,114.7,114.5\left(\mathrm{~d},{ }^{2} J_{\mathrm{C}-\mathrm{F}}=\right.$ $22.0 \mathrm{~Hz}), 111.1,110.9,69.9$; IR (KBr) v: 3550, 3416, 2062, 1638, 1617, 1487, 1396, 1174, 787, $622 \mathrm{~cm}^{-1}$; HRMS calcd for $\mathrm{C}_{34} \mathrm{H}_{23} \mathrm{ClFN}_{6} \mathrm{O}_{3}[\mathrm{M}+\mathrm{H}]^{+}:$617.1504, found 617.1512.

$(E)-3-(((E)-(5-(4-(3-$ 氯-4-(3-氟苄氧基)苯胺基)喹唑 啉-6-基)呋喃-2-基)亚甲基)亚肼基)-5-氟吲哚啉-2-酮 (4h): 红色固体 $0.23 \mathrm{~g}$, 收率为 $72.1 \%$. m.p. $>280{ }^{\circ} \mathrm{C} ;{ }^{1} \mathrm{H}$ NMR (600 MHz, DMSO- $\left.d_{6}\right) \delta: 10.85(\mathrm{~s}, 1 \mathrm{H}), 9.99$ (s, 1H), 8.95 (s, $1 \mathrm{H}), 8.66$ (s, 1H), 8.58 (s, $1 \mathrm{H}), 8.24$ (d, $J=8.6 \mathrm{~Hz}$, $1 \mathrm{H}), 8.08$ (dd, $J=8.3,2.4 \mathrm{~Hz}, 1 \mathrm{H}), 8.01(\mathrm{~d}, J=1.8 \mathrm{~Hz}$, $1 \mathrm{H}), 7.82(\mathrm{~d}, J=8.6 \mathrm{~Hz}, 1 \mathrm{H}), 7.76(\mathrm{dd}, J=9.0,1.8 \mathrm{~Hz}$, $1 \mathrm{H}), 7.57(\mathrm{~d}, J=3.5 \mathrm{~Hz}, 1 \mathrm{H}), 7.50 \sim 7.46(\mathrm{~m}, 1 \mathrm{H}), 7.40(\mathrm{~d}$, $J=3.5 \mathrm{~Hz}, 1 \mathrm{H}), 7.35 \sim 7.31(\mathrm{~m}, 2 \mathrm{H}), 7.26(\mathrm{~d}, J=9.0 \mathrm{~Hz}$, $1 \mathrm{H}), 7.23(\mathrm{dd}, J=8.5,2.5 \mathrm{~Hz}, 1 \mathrm{H}), 7.19(\mathrm{~d}, J=8.9 \mathrm{~Hz}$, $1 \mathrm{H}), 6.88(\mathrm{dd}, J=8.5,4.2 \mathrm{~Hz}, 1 \mathrm{H}), 5.25(\mathrm{~s}, 2 \mathrm{H}) ;{ }^{13} \mathrm{C} \mathrm{NMR}$ $\left(151 \mathrm{MHz}\right.$, DMSO- $\left.d_{6}\right) \delta: 164.8,162.2\left(\mathrm{~d},{ }^{1} J_{\mathrm{C}-\mathrm{F}}=243.6\right.$ $\mathrm{Hz})$, 158.4, 157.6, 157.0, 155.9 (d, $\left.{ }^{1} J_{\mathrm{C}-\mathrm{F}}=265.2 \mathrm{~Hz}\right)$, $152.3,151.7,149.8,149.7,149.1,141.1,139.6\left(\mathrm{~d},{ }^{3} J_{\mathrm{C}-\mathrm{F}}=\right.$
$7.9 \mathrm{~Hz}), 132.9,130.5\left(\mathrm{~d},{ }^{3} J_{\mathrm{C}-\mathrm{F}}=8.4 \mathrm{~Hz}\right), 128.8\left(\mathrm{~d},{ }^{2} J_{\mathrm{C}-\mathrm{F}}=\right.$ $30.9 \mathrm{~Hz}), 126.6,124.2,123.3\left(\mathrm{~d},{ }^{4} J_{\mathrm{C}-\mathrm{F}}=2.6 \mathrm{~Hz}\right), 122.9$, $122.4,121.1,120.0\left(\mathrm{~d},{ }^{4} J_{\mathrm{C}-\mathrm{F}}=2.6 \mathrm{~Hz}\right), 119.9\left(\mathrm{~d},{ }^{2} J_{\mathrm{C}-\mathrm{F}}=\right.$ $24.1 \mathrm{~Hz}), 119.1,117.2\left(\mathrm{~d},{ }^{3} J_{\mathrm{C}-\mathrm{F}}=9.6 \mathrm{~Hz}\right), 116.3,115.3$, $114.7\left(\mathrm{~d},{ }^{2} J_{\mathrm{C}-\mathrm{F}}=21.1 \mathrm{~Hz}\right), 114.2,114.0\left(\mathrm{~d},{ }^{2} J_{\mathrm{C}-\mathrm{F}}=21.9 \mathrm{~Hz}\right)$, $111.5\left(\mathrm{~d},{ }^{3} J_{\mathrm{C}-\mathrm{F}}=7.4 \mathrm{~Hz}\right), 110.4,69.4$; IR $(\mathrm{KBr}) v: 3549$, 3416, 3235, 2062, 1638, 1617, 1488, 1396, 1174, 623 $\mathrm{cm}^{-1}$; HRMS calcd for $\mathrm{C}_{34} \mathrm{H}_{22} \mathrm{ClF}_{2} \mathrm{~N}_{6} \mathrm{O}_{3}[\mathrm{M}+\mathrm{H}]^{+}$: 635.1410, found 635.1418.

(E)-3-(( $E)-(5-(4-(3-$ 氯-4-(3-氟苄氧基)苯胺基)喹唑 啉-6-基)呋喃-2-基)亚甲基)亚肼基)-5-氯吲哚啉-2-酮(4i): 红色固体 $0.26 \mathrm{~g}$, 收率为 $78.7 \%$. m.p. $>280{ }^{\circ} \mathrm{C} ;{ }^{1} \mathrm{H}$ NMR $\left(600 \mathrm{MHz}, \mathrm{DMSO}-d_{6}\right) \delta: 10.93(\mathrm{~s}, 1 \mathrm{H}), 9.93(\mathrm{~s}, 1 \mathrm{H}), 8.92$ $(\mathrm{s}, 1 \mathrm{H}), 8.66(\mathrm{~s}, 1 \mathrm{H}), 8.55(\mathrm{~s}, 1 \mathrm{H}), 8.36(\mathrm{~s}, 1 \mathrm{H}), 8.24(\mathrm{~d}$, $J=8.7 \mathrm{~Hz}, 1 \mathrm{H}), 7.97$ (s, 1H), $7.78(\mathrm{~d}, J=8.7 \mathrm{~Hz}, 1 \mathrm{H}), 7.72$ $(\mathrm{d}, J=8.0 \mathrm{~Hz}, 1 \mathrm{H}), 7.52(\mathrm{~d}, J=3.4 \mathrm{~Hz}, 1 \mathrm{H}), 7.50 \sim 7.46$ (m, $1 \mathrm{H}), 7.39(\mathrm{~d}, J=8.5 \mathrm{~Hz}, 1 \mathrm{H}), 7.37 \sim 7.32(\mathrm{~m}, 3 \mathrm{H})$, $7.26(\mathrm{~d}, J=8.9 \mathrm{~Hz}, 1 \mathrm{H}), 7.19$ (t, $J=8.9 \mathrm{~Hz}, 1 \mathrm{H}), 6.87$ (d, $J=8.3 \mathrm{~Hz}, 1 \mathrm{H}), 5.26(\mathrm{~s}, 2 \mathrm{H}) ;{ }^{13} \mathrm{C}$ NMR $(151 \mathrm{MHz}$, DMSO- $\left.d_{6}\right) \delta: 164.5,162.2\left(\mathrm{~d},{ }^{1} J_{\mathrm{C}-\mathrm{F}}=243.7 \mathrm{~Hz}\right), 157.7$, $157.0,155.0,152.5,151.6,149.8,148.9,143.4,139.6(\mathrm{~d}$, $\left.{ }^{3} J_{\mathrm{C}-\mathrm{F}}=7.4 \mathrm{~Hz}\right), 132.9,132.7,130.5\left(\mathrm{~d},{ }^{3} J_{\mathrm{C}-\mathrm{F}}=8.4 \mathrm{~Hz}\right)$, $129.2,128.6\left(\mathrm{~d},{ }^{2} J_{\mathrm{C}-\mathrm{F}}=24.2 \mathrm{~Hz}\right), 126.5,126.0,124.3$, $123.4,123.3\left(\mathrm{~d},{ }^{4} J_{\mathrm{C}-\mathrm{F}}=2.6 \mathrm{~Hz}\right), 122.5,121.0,119.2,118.0$, $115.3,114.7\left(\mathrm{~d},{ }^{2} J_{\mathrm{C}-\mathrm{F}}=20.8 \mathrm{~Hz}\right), 114.3,114.2,114.1$, 113.9, 112.0, 110.3, 69.4; IR (KBr) v: 3551, 3417, 2062, 1617, 1487, 1397, 1174, 1002, 787, $622 \mathrm{~cm}^{-1}$; HRMS calcd for $\mathrm{C}_{34} \mathrm{H}_{22} \mathrm{Cl}_{2} \mathrm{FN}_{6} \mathrm{O}_{3}[\mathrm{M}+\mathrm{H}]^{+}:$651.1114, found 651.1118 .

\subsection{3 抗肿瘤活性实验}

化合物 $4 \mathbf{a} \sim 4 \mathbf{i}$ 对肿瘤细胞的生长抑制活性利用 MTT 法进行测定. 分别取对数生长期的人肿瘤细胞, 用 胰蛋白酶消化液消化、离心、重悬后计数, 调整细胞悬 液浓度为 $2.0 \times 10^{4} \sim 5.0 \times 10^{4}$ 个 $/ \mathrm{mL}$ ，随后接种于 96 孔 细胞培养板中 $\left(100 \mu \mathrm{L} /\right.$ 孔), 置饱和湿度、 $37{ }^{\circ} \mathrm{C}$ 和 $5 \%$ $\mathrm{CO}_{2}$ 培养箱中培养 $24 \mathrm{~h}$. 受试化合物用培养基稀释至所 需浓度, 加入已接种人肿瘤细胞的细胞培养板中 $(100$ $\mu \mathrm{L} /$ 孔), DMSO 终浓度为 $0.5 \%$, 置于培养箱中继续培养. $72 \mathrm{~h}$ 后, 加入 MTT $(20 \mu \mathrm{L} /$ 孔), 培养箱中反应 $4 \mathrm{~h}$. 吸弃 孔内液体, 加入 DMSO (150 $\mu \mathrm{L} /$ 孔), 摇床上震荡 $10 \mathrm{~min}$. 待甲肤完全溶解后, 用酶标仪测定 $570 \mathrm{~nm}$ 波长处的吸 光度 (OD 值), $630 \mathrm{~nm}$ 波长处的吸光度作为参比, 以相应 溶剂作为对照, 计算细胞生长抑制率.

受试化合物对肿瘤细胞生长抑制率的计算公式: 抑 制率 $(\%)=\left[1-\left(\mathrm{OD}_{\mathrm{S}}-\mathrm{OD}_{\mathrm{NC}}\right) /\left(\mathrm{OD}_{\mathrm{PC}}-\mathrm{OD}_{\mathrm{NC}}\right)\right] \times 100 \%$. 
其中: $\mathrm{OD}_{\mathrm{S}}$ 表示样品孔的吸光度值(细胞十待测化合物十 $\mathrm{MTT}) ; \mathrm{OD}_{\mathrm{PC}}$ 表示对照孔的吸光度值 $($ 细胞 $+\mathrm{DMSO}+$ $\mathrm{MTT}) ; \mathrm{OD}_{\mathrm{NC}}$ 表示调零孔的吸光度值(培养基 $+\mathrm{DMSO}+$ $\mathrm{MTT})$. $\mathrm{OD}_{\mathrm{S}}=\mathrm{OD}_{570 \mathrm{~S}}-\mathrm{OD}_{630 \mathrm{~S}} ; \mathrm{OD}_{\mathrm{PC}}=\mathrm{OD}_{570 \mathrm{PC}}-\mathrm{OD}_{630 \mathrm{PC}}$; $\mathrm{OD}_{\mathrm{NC}}=\mathrm{OD}_{570 \mathrm{NC}}-\mathrm{OD}_{630 \mathrm{NC}}$.

受试化合物对肿瘤细胞生长抑制曲线的拟合及 $\mathrm{IC}_{50}$ 的计算: 采用 GraphpadPrism5 拟合受试化合物对肿瘤细 胞生长的抑制曲线, 并得出 $\mathrm{IC}_{50}$ 值. 每组设置 3 个复孔, 至少重复 3 次.

\section{辅助材料(Supporting Information) 化合物 $\mathbf{4 a} \sim \mathbf{4 i}$ 的} NMR, HRMS, IR 图谱和晶体数据的 PDF 文件. 这些材 料可以免费从本刊网站(http://sioc-journal.cn/)上下载.

\section{Referenes}

[1] Lipunova, G. N.; Nosova, E. V.; Charushin, V. N.; Chupakhin, O. N. Russ. Chem. Rev. 2016, 85, 759 .

[2] Gordana, V.; Jeffrey, C. Oncologist 2003, 8, 531.

[3] Ioannou, N.; Seddon, A. M.; Dalgleish, A.; Mackintosh, D.; Solca, F.; Modjtahedi, H. Int. J. Oncol. 2016, 48, 908.

[4] Ravaud, A.; Hawkins, R.; Gardner, J. P.; Vonder Maase, H.; Zantl, N.; Harper, P.; Rolland, F.; Audhuy, B.; Machiels, J. P.; Petavy, F.; Gore, M.; Schoffski, P.; El-Hariry, I. J. Clin. Oncol. 2008, 26, 2285.

[5] El-Ansary, A. K.; Kamal, A. M.; Abd-Hafiz, A. G. M. Chem. Pharm. Bull. 2016, 64, 1172.

[6] Wang, W.-J.; Li, J; Zhang, Y.-L.; Zhang, X.-Q.; Gu, H.-M.; Li, B.-L. Chem. Bull 2015, 78, 1017 (in Chinese). (王维佳, 李江, 张娅玲, 张喜全, 顾红梅, 李宝林, 化学通报, 2015, 78, 1017.)

[7] Nahta, R.; Yuan, L. X. H.; Du, Y.; Esteva, F. J. Mol. Cancer Ther. 2007, 6, 667 .
[8] Lee, D.; Long, S. A.; Murray, J. H.; Adams, J. L.; Nuttall, M. E.; Nadeau, D. P.; Kikly, K.; Winkler, J. D.; Sung, C. M.; Ryan, M. D.; Levy, M. A.; Keller, P. M.; DeWolf, W. E. Jr. J. Med. Chem. 2001, 44, 2015.

[9] Chapman, J. G.; Magee, W. P.; Stukenbrok, H. A.; Beckius, G. E.; Milici, A. J.; Ross Tracey, W. Eur. J. Pharmacol. 2002, 456, 59.

[10] Smith, B. D.; Smith, G. L.; Hurria, A.; Hortobagyi, G. N.; Buchholz, T. A. Clin. Oncol. 2009, 27, 2758.

[11] Atkins, M.; Jones, C. A.; Kirkpatrick, P. Nat. Rev. Drug Discovery 2006, 5,279 .

[12] Wang, J.; Zhu, C.-Y.; Guo, J.-Y.; Zhang, Z.-G.; Chen, F.; Zhang, L. Guangdong Chem. Ind. 2015, 42, 106 (in Chinese). (王京，朱春媛，国佳莹，张泽国，陈凡，张否，广东化工，2015, 42, 106.)

[13] Hu, C.; Solomon, V. R.; Cano, P.; Lee, H. Eur. J. Med. Chem. 2010, 45,705 .

[14] Sella, A.; Yarom, N.; Zisman, A.; Kovel, S. Oncology 2009, 76, 442.

[15] Solomon, V. R.; Hu, C.; Lee, H. Bioorg. Med. Chem. 2009, 17, 7585.

[16] Fares, M.; Eldehna, W. M.; Abou-Seri, S. M.; Abdel-Aziz, H. A.; Aly, M. H.; Tolba, M. F. Arch. Pharm. 2015, 348, 144.

[17] Ji, X.; Wang, W.-W.; Xu, G.-H.; Li, F.; Yao, S.-C. Chin. J. Pharm. 2009, 40, 801 (in Chinese)

(季兴, 王武伟，许贯虹，李飞，姚社春，中国医药工业杂志， $\mathbf{2 0 0 9}, 40,801$.)

[18] Liu, J; Zhang, Y.-L.; Zhang, X.-Q.; Gu, H.-M.; Li, B.-L. Chem. Bull. 2016, 79, 360 (in Chinese) (刘娟，张娅玲，张喜全，顾红梅，李宝林，化学通报，2016，79， 360.)

[19] McClure, M. S.; Osterhout, M. H.; Roschangar, F.; Sacchetti, M. J. WO 2002002552, 2002 [Chem. Abstr. 2002, 136, 102396].

[20] Hassan, T. A. F. M.; Kadi, A. A.; Abdel-Aziz, H. A. K. US 20120252860, 2012 [Chem. Abstr. 2012, 157, 548485].

[21] Dweedar, H. E.; Mahrous, H.; Ibrahim, H. S.; Abdel-Aziz, H. A. Eur. J. Med. Chem. 2014, 78, 275.

[22] Nikalje, A. P.; Ansari, A.; Bari, S.; Ugale, V. Arch. Pharm. 2015 , $348,433$. 